\title{
On the properties of a class of higher-order Mathieu equations originating from a parametric quantum oscillator
}

\author{
Subhadip Biswas $(\mathbb{D}$ • Jayanta K. Bhattacharjee
}

Received: 9 August 2018 / Accepted: 31 January 2019 / Published online: 26 February 2019

(C) The Author(s) 2019

\begin{abstract}
Evolution in time of an arbitrary initial state for a parametrically driven quantum oscillator is an interesting problem since there exist regions in parameter space (defined by the amplitude and frequency of the driving) where the moments of the probability distribution can diverge in time. While the first moment satisfies a Mathieu equation, the higher-order moments follow Mathieu like equations of order greater than two. It is not very often that a physical problem gives rise to higher-order Mathieu equations. Hence, we give a detailed study of the different stability zones associated with the parametric quantum oscillator, using perturbative techniques traditionally associated with the Mathieu equation. We verify our results by numerical analysis, thus demonstrating that for the higher-order Mathieu equations, the traditional perturbation theory methods give a consistent account of the stability zones.
\end{abstract}

Keywords Higher-order Mathieu · KrylovBogoliubov · Quantum parametric oscillator

\footnotetext{
S. Biswas $(\bowtie)$

Department of Physics and Astronomy, University of Sheffield, Sheffield S3 7RH, UK

e-mail: sbiswas2@sheffield.ac.uk

J. K Bhattacharjee

Department of Theoretical Physics, Indian Association for the Cultivation of Science, Jadavpur, Kolkata 700032, India e-mail: tpjkb@iacs.res.in
}

\section{Introduction}

Mathieu equations describe parametrically driven oscillators. They have been investigated for decades using a variety of techniques like harmonic balance, LindstedtPoincare perturbation theory, multiple timescale techniques and Krylov-Bogoliubov amplitude and phase equations. An important feature of the Mathieu equation is that systems described by it show instability in a set of tongue-shaped regions in the parameter space spanned by the driving frequency and the amplitude of the drive. The tongues emanate from certain points on the frequency axis where the natural frequency is $\frac{n}{2}$ times the driving frequency with $n=1,2,3 \ldots$ being the set of integers. The motion is strictly periodic only on the lines separating the stable from the unstable zones. In the stable zone, the motion is quasiperiodic in general. The techniques referred to above are used to study the boundaries where the motion is periodic. These techniques have been covered comprehensively in various texts and lecture notes (e.g. [1-3]). The study of coupled Mathieu equations became popular about three decades ago and can be found in the treatment of elastic pendula (two coupled degrees of freedom) [4], in the study of colliding particle beams in a two-dimensional accelerator [5] or in the description of two-mode dynamics of rotating rings with variable spin-speed [6]. The fact that the usual techniques for obtaining the boundaries of the single- variable Mathiеu equation can be used to calculate the unstable zones 
of the coupled system was demonstrated clearly by Mahmoud [7].

More recently, a different perspective on this class of problems was discussed by Landa et al. [8]. While the usual emphasis had been on finding the instability zones and the periodic solutions on the boundary of the instability zones, these authors developed a technique for finding the classical as well as the quantum solutions in the region where the motion is bounded. One of the important problems addressed in this work is the one-dimensional quantum parametric oscillator which describes the motion of a single ion confined in a radio-frequency trap [9]. A comprehensive account of the existence of periodic orbits for a variety of generalised forms of the Mathieu equation was provided by Younesian et al. [10]. This included nonlinear Mathіеu equations treated by harmonic balance and averaging [11], bifurcations in cubic Mathieu equations [12] and resonances in quasi-periodic Mathieu equations [13]. Another direction in which the Mathieu equation was extended was the introduction of time delay [14]. That the usual perturbative techniques associated with the Mathieu equation could be used for a nonlinear Mathieu equation with delay was established by Morrison and Rand [15]. Yet another extension of these driven systems has been the introduction of systems with fractional derivatives. The appropriateness of the usual perturbative techniques for exploring the regions of bounded motion has recently been established [16$18]$.

While the second-order (recently even fractional order) non-autonomous differential equations, which is the class to which the Mathieu equation belongs, have been studied from a formal mathematical standpoint as well as from the applicational viewpoint of perturbation theory, the higher-order equations have been, to the best of our knowledge, studied only from a formal perspective [19-22]. It turns out that the one-dimensional quantum parametric oscillator discussed above gives rise to an infinite set of higher-order Mathieu like equations. Our aim in this paper will be to explore the effectiveness of the usual perturbative techniques to study the unstable zones of these higher-order Mathieu-like equations. We will use known perturbative techniques to find the boundaries between the regions where the solution is bounded and where they are unbounded. It will turn out that the boundaries thus obtained will agree with the boundaries obtained numerically.
A combination of Heisenberg's equation of motion and Ehrenfest's theorem in quantum mechanics describes the time development of the expectation value of any operator $\mathcal{O}(x)$ under a given Hamiltonian $\mathcal{H}$. If $\Psi(x, t)$ is the wave function at any time $t$ (we consider only one- dimensional space), then the expectation value is $\langle\mathcal{O}\rangle=\int_{-\infty}^{\infty} \Psi^{*}(x, t) \mathcal{O}(x) \Psi(x, t) \mathrm{d} x$ and Ehrenfest's theorem states

$i \hbar \frac{\mathrm{d}}{\mathrm{d} t}\langle\mathcal{O}\rangle=\langle[\mathcal{O}, \mathcal{H}]\rangle$

where $\langle[\mathcal{O}, \mathcal{H}]\rangle$ is the commutator of $\mathcal{O}$ and $\mathcal{H}$. A quantum mechanical state is prescribed by $\Psi(x, t)$ which is a complex valued function generally written as $\Psi(x, t)=\sqrt{P(x, t)} e^{i \phi(x, t)}$, where $P(x, t)=$ $|\Psi(x, t)|^{2}$ is the probability distribution associated with the state at any time $t$. Ehrenfest's theorem has rarely been used except to study classical trajectories. However, it can provide useful information about various moments of the probability distribution and as such can yield information regarding the form of the distribution. A particularly interesting situation is the motion of a particle in a time-dependent harmonic trapping function represented by the potential $\mathcal{V}=\frac{1}{2} \mathrm{~m} \omega^{2} x^{2}(1+\epsilon f(t))$ where $f(t)$ is a periodic function with period $T=\frac{2 \pi}{\Omega}$. The Hamiltonian operator for the quantum parametric oscillator is [23]

$\mathcal{H}=\frac{p^{2}}{2 m}+\frac{1}{2} \mathrm{~m} \omega^{2} x^{2}(1+\epsilon f(t))$

with $f(t)=f(t+T)=f\left(t+\frac{2 \pi}{\Omega}\right)$. In the above, $p=-i \hbar \frac{\mathrm{d}}{\mathrm{d} x}$ is the momentum operator. In this case, for an initial wave function which is Gaussian, the time development of $\Psi(x, t)$ can be found if the dynamics is in the part of $\omega-\epsilon$ space where the classical motion is bounded [24-27]. For arbitrary initial wave functions, it is virtually impossible to arrive at the time development exactly. In this situation, Ehrenfest's theorem is very useful since it provides exact equations for all the moments. Each moment gives rise to a nonautonomous dynamical [28] system, and this very physical problem gives rise to the whole set of interesting mathematical systems which are worth studying in their own right. In this work, we will focus on the mathematical aspects of the dynamical systems arising from Ehrenfest's theorem for the moments.

The process of arriving at the equations of motion is straightforward. One needs to use Eq. (1) and carry out evaluation of $[\mathcal{O}, \mathcal{H}]$ with the $\mathcal{H}$ of Eq. (2). As an example, we explicitly work out the first moment $\langle x\rangle$ 
which represents the mean position of the quantum particle. From Eq. (1) and the basic commutation relation $[x, p]=i \hbar$, we see that

$$
\begin{aligned}
\frac{\mathrm{d}}{\mathrm{d} t}\langle x\rangle & =\frac{1}{i \hbar}\left\langle\left[x, \frac{p^{2}}{2 m}+\frac{1}{2} \mathrm{~m} \omega^{2} x^{2}(1+\epsilon f(t))\right]\right\rangle \\
& =\frac{1}{i \hbar}\left\langle\left[x, \frac{p^{2}}{2 m}\right]\right\rangle=\frac{\langle p\rangle}{m}
\end{aligned}
$$

and

$$
\begin{aligned}
\frac{\mathrm{d}}{\mathrm{d} t}\langle p\rangle & =\frac{1}{i \hbar}\left\langle\left[p, \frac{p^{2}}{2 m}+\frac{1}{2} \mathrm{~m} \omega^{2} x^{2}(1+\epsilon f(t))\right]\right\rangle \\
& =\frac{1}{i \hbar} \frac{1}{2} \mathrm{~m} \omega^{2}\left\langle\left[p, x^{2}(1+\epsilon f(t))\right]\right\rangle \\
& =-\mathrm{m} \omega^{2}\langle x\rangle(1+\epsilon f(t))
\end{aligned}
$$

One move derivative of Eq. (3) and use of Eq. (4) leads to

$$
\frac{\mathrm{d}^{2}}{\mathrm{~d} t^{2}}\langle x\rangle+\omega^{2}(1+\epsilon f(t))\langle x\rangle=0
$$

This is the dynamics of the mean position of the particle, and it follows the classical trajectory obtained from the Hamiltonian of Eq. (2).

If $f(t)=\cos (\Omega t)$, then we have the familiar Mathieu equation.

$$
\frac{\mathrm{d}^{2}}{\mathrm{~d} t^{2}}\langle x\rangle+\omega^{2}(1+\epsilon \cos (\Omega t))\langle x\rangle=0
$$

The dynamics of all moments $\left\langle x^{n}\right\rangle$ have to be obtained by a process of repeated differentiation and use of Eq. (1). The moments $\left\langle x^{n}\right\rangle$ are associated with different features of the probability distribution. In particular for $n=2$,

$V=\left\langle x^{2}\right\rangle-\langle x\rangle^{2}$

is the variance. This is the most important moment as its nonzero value signals a complete departure from classical physics. For $n=3$, we have

$S=\left\langle(x-\langle x\rangle)^{3}\right\rangle$,

which is the skewness, and for $n=4$

$K=\left\langle(x-\langle x\rangle)^{4}\right\rangle$,

which is the kurtosis of the distribution. We write down the equations of motion (the derivation of the dynamics of $V$ is shown in "Appendix A", the others follow in a similar manner)

$$
\frac{\mathrm{d}^{3}}{\mathrm{~d} t^{3}} V+4 \omega^{2}(1+\epsilon f(t)) \frac{\mathrm{d}}{\mathrm{d} t} V+2 \omega^{2} \epsilon \dot{f} V=0
$$

$$
\begin{aligned}
& \frac{\mathrm{d}^{4}}{\mathrm{~d} t^{4}} S+10 \omega^{2}(1+\epsilon f(t)) \frac{\mathrm{d}^{2}}{\mathrm{~d} t^{2}} S+9 \omega^{4}(1+\epsilon f(t))^{2} S \\
& +10 \omega^{2} \epsilon \dot{f} \frac{\mathrm{d}}{\mathrm{d} t} S+3 \omega^{2} \epsilon \ddot{f} S=0
\end{aligned}
$$

and

$$
\begin{aligned}
& \frac{\mathrm{d}^{5}}{\mathrm{~d} t^{5}} K+20 \omega^{2}(1+\epsilon f(t)) \frac{\mathrm{d}^{3}}{\mathrm{~d} t^{3}} K+24 \omega^{2} \epsilon \dot{f} \frac{\mathrm{d}^{2}}{\mathrm{~d} t^{2}} K \\
& \quad+64 \omega^{4}(1+\epsilon f(t))^{2} \frac{\mathrm{d}}{\mathrm{d} t} K+18 \omega^{2} \epsilon \ddot{f} \frac{\mathrm{d}}{\mathrm{d} t} K \\
& \quad+64 \omega^{4} \epsilon(1+\epsilon f(t)) \dot{f} K+4 \omega^{2} \epsilon \dddot{f} K=0
\end{aligned}
$$

For the discussions in this paper, we will focus entirely on $f(t)=\cos (\Omega t)$.

The properties of Mathieu equation (Eq. 6) are well known [1-3]. We will recall them in Sect. 2 to carefully explain the perturbation theory technique that we will use and the numerical procedure that we will follow in the rest of the paper.

If we consider the dynamics of the variance $V$ and redefine $4 \omega^{2}$ as $\bar{\omega}^{2}$, then Eq. (10) becomes for $f(t)=$ $\cos (\Omega t)$

$$
\begin{gathered}
\frac{\mathrm{d}^{3} V}{\mathrm{~d} t^{3}}+\bar{\omega}^{2}(1+\epsilon \cos (\Omega t)) \frac{\mathrm{d} V}{\mathrm{~d} t} \\
-\frac{\epsilon \bar{\omega}^{2}}{2} \Omega \sin (\Omega t) V=0
\end{gathered}
$$

We further simplify by redefining the combination $\epsilon \bar{\omega}^{2}$ as $\bar{\epsilon}$, so that

$$
\begin{gathered}
\frac{\mathrm{d}^{3} V}{\mathrm{~d} t^{3}}+\bar{\omega}^{2} \frac{\mathrm{d} V}{\mathrm{~d} t}+\bar{\epsilon} \cos (\Omega t) \frac{\mathrm{d} V}{\mathrm{~d} t} \\
-\frac{\bar{\epsilon}}{2} \Omega \sin (\Omega t) V=0
\end{gathered}
$$

The above equation corresponds to the dynamical system with the structure $X_{i}=A_{i j}(t) X_{j}$ with $A_{i j}(t)=$ $A_{i j}\left(t+\frac{2 \pi}{\Omega}\right)=A_{i j}(t+T)$.

Explicitly,

$$
\begin{aligned}
\dot{V} & =U \\
\dot{U} & =W \\
\dot{W} & =-\bar{\omega}^{2} U-\bar{\epsilon} \cos (\Omega t) U+\frac{\bar{\epsilon}}{2} \Omega \sin (\Omega t) V
\end{aligned}
$$

The trace of the matrix $A_{i j}(t)$ is clearly zero, and hence, the three Floquet multipliers $\mu_{1}, \mu_{2}$ and $\mu_{3}$ satisfy $\mu_{1} \mu_{2} \mu_{3}=1$. Consequently, the periodic solutions can have the periods $T, 2 T$ and $3 T$. For a subharmonic response of period $3 T$ (frequency $\frac{\Omega}{3}$ ), we would have periodic solutions originating from $\bar{\omega}=\frac{\Omega}{3}, \frac{4 \Omega}{3} \ldots$, etc. For a periodic response of period $2 T$, the solutions 
start at $\bar{\omega}=\frac{\Omega}{2}, \frac{3 \Omega}{2} \ldots$, etc and for a response of period $T$, the solutions start at $\bar{\omega}=\Omega, 2 \Omega \ldots$, etc.

In a similar fashion, the equations of motion of $S$ and $K$ are in principle capable of showing periodicities $T, 2 T, 3 T, 4 T$ and $T, 2 T, 3 T, 4 T, 5 T$, respectively.

Using different perturbation techniques [2], we will study the properties of the dynamical systems shown in Eqs. (10)-(12). The techniques (harmonic balance and Krylov-Bogoliubov) will be geared to finding the periodic trajectories. In the case of Eq. (13) specifically, we obtain the following:

- A) Harmonic balance shows that period $3 T$ trajectories exist only on a curve emanating from $\bar{\omega}=\frac{\Omega}{3}$ with no instability zones in the vicinity of $\frac{\Omega}{3}$.

- B) A similar situation holds in the vicinity of $\bar{\omega}=$ $\frac{\Omega}{2}$, although the structure of Eq. (13) would have indicated otherwise.

- C) In the vicinity of $\bar{\omega}=\Omega$, the KrylovBogoliubov technique reveals the existence of two curves corresponding to the periodic orbits emanating from this point. In the region bounded by the two curves lies the instability zone where the solutions are unbounded. The boundaries, quite surprisingly, happen to be identical to that for Eq. (6) a result confirmed by our numerical studies.

For Eq. (11), the conclusions are qualitatively similar with the unstable zone emanating from $\bar{\omega}=\Omega$ alone. For Eq. (12), however, we have instability zones emanating from $\bar{\omega}=\frac{\Omega}{4}, \frac{\Omega}{2}$ and $\Omega$. In this case, the instability zone emanating from $\bar{\omega}=\Omega$ is far wider than those for Eqs. (6), (10) and (11).

We briefly recapitulate the primary results of the Mathieu equation in Sect. 2. In Sect. 3, we deal with the dynamics of the variance in detail, and in Sect. 4, we study the skewness and the kurtosis. We conclude with a short summary in Sect. 5 .

\section{Properties of Mathieu equation}

In this section, we review the properties of the Mathieu equation which is a second-order differential equation

$\ddot{X}+\omega^{2} X+\epsilon \cos (\Omega t) X=0$.

It has the dynamical system form $\dot{X}=Y$ and $\dot{Y}=$ $-\left(\omega^{2}+\epsilon \cos (\Omega t) X\right)$. Since the dynamical system is traceless, the two Floquet multipliers $\mu_{1,2}$ satisfy $\mu_{1} \mu_{2}=1$. This means that the system can have responses of period $T$ and $2 T$. Since $T=\frac{\Omega}{2 \pi}$, harmonic balance indicates that period $2 T$ solutions should emerge from $\omega=\frac{\Omega}{2}$ and period $T$ solution should emerge from $\omega=\Omega$. We first explore the straightforward perturbative method for obtaining periodic solutions of period $2 T$, around $\omega=\frac{\Omega}{2}$. Accordingly, we write

$\omega=\frac{\Omega}{2}+\Delta \omega(\epsilon)=\frac{\Omega}{2}+\delta_{1} \epsilon+\delta_{2} \epsilon^{2}+\cdots$

and expand

$X=X_{0}+X_{1} \epsilon+X_{2} \epsilon^{2}+\cdots$

Substituting into Eq. (16) and equating the coefficient of each power of $\epsilon$ to zero, we have

$\ddot{X}_{0}+\frac{\Omega^{2}}{4} X_{0}=0$

$\ddot{X}_{1}+\frac{\Omega^{2}}{4} X_{1}=-\Omega \delta_{1} X_{0}-\cos (\Omega t) X_{0}$

$\ddot{X}_{2}+\frac{\Omega^{2}}{4} X_{2}=-\Omega \delta_{2} X_{0}-\delta_{1}^{2} X_{0}-\Omega \delta_{1} X_{1}$

$-\cos (\Omega t) X_{0}$

From Eq. (19a), $X_{0}=A \cos \left(\frac{\Omega}{2} t\right)+B \sin \left(\frac{\Omega}{2} t\right)$ and inserting this solution in Eq. (19b),

$$
\begin{gathered}
\ddot{X}_{1}+\frac{\Omega^{2}}{4} X_{1}=-\Omega \delta_{1}\left(A \cos \left(\frac{\Omega}{2} t\right)+B \sin \left(\frac{\Omega}{2} t\right)\right) \\
-\cos (\Omega t x)\left(A \cos \left(\frac{\Omega}{2} t\right)+B \sin \left(\frac{\Omega}{2} t\right)\right) \\
=-\Omega \delta_{1} A \cos \left(\frac{\Omega}{2} t\right)-\Omega \delta_{1} B \sin \left(\frac{\Omega}{2} t\right) \\
-\frac{A}{2}\left(\cos \left(\frac{3 \Omega}{2} t\right)+\cos \left(\frac{\Omega}{2} t\right)\right) \\
-\frac{B}{2}\left(\sin \left(\frac{3 \Omega}{2} t\right)-\sin \left(\frac{\Omega}{2} t\right)\right)
\end{gathered}
$$

For $X_{1}$ to be finite, we cannot have $\cos \left(\frac{\Omega t}{2}\right)$ or $\sin \left(\frac{\Omega t}{2}\right)$ terms on the right-hand side. These terms are known as secular terms and have to be removed order by order in perturbation theory. This requires $B=0$ and $\delta_{1}=-\frac{1}{2 \Omega}$ if the solutions are of the $\cos \left(\frac{\Omega t}{2}\right)$ variety and $A=0$ and $\delta_{1}=+\frac{1}{2 \Omega}$ if the solutions are of the form $\sin \left(\frac{\Omega t}{2}\right)$. Thus, the curves on which the solution is periodic are given by

$X=A \cos \left(\frac{\Omega t}{2}\right) \quad$ on $\omega=\frac{\Omega}{2}-\frac{\epsilon}{2 \Omega}+\mathcal{O}\left(\epsilon^{2}\right)$ 


$$
X=B \sin \left(\frac{\Omega t}{2}\right) \quad \text { on } \omega=\frac{\Omega}{2}+\frac{\epsilon}{2 \Omega}+\mathcal{O}\left(\epsilon^{2}\right)
$$

That the solution is divergent between the two lines of periodicity above and quasi-periodic outside is shown by the technique of Krylov-Bogoliubov. It proceeds by writing the solution $X(t)$ as

$$
X(t)=A(t) \cos \left(\frac{\Omega t}{2}\right)+B(t) \sin \left(\frac{\Omega t}{2}\right)
$$

near the point $\omega=\frac{\Omega}{2}$. We will assume $A(t)$ and $B(t)$ to be slowly varying (so that $\dot{A}<<\ddot{A}$ and similarly for $B$ ) and find the dynamical systems for $A$ and $B$. The fixed point will give the periodic solutions. Taking derivatives and keeping the relevant terms

$$
\begin{aligned}
\dot{X}(t)= & \dot{A}(t) \cos \left(\frac{\Omega t}{2}\right)+\dot{B}(t) \sin \left(\frac{\Omega t}{2}\right) \\
& +\frac{\Omega}{2} B(t) \cos \left(\frac{\Omega t}{2}\right)-\frac{\Omega}{2} A(t) \sin \left(\frac{\Omega t}{2}\right) \\
\ddot{X}(t)= & -\Omega \dot{A}(t) \sin \left(\frac{\Omega t}{2}\right)+\Omega \dot{B}(t) \cos \left(\frac{\Omega t}{2}\right) \\
& -\frac{\Omega^{2}}{4}\left(A(t) \cos \left(\frac{\Omega t}{2}\right)+B(t) \sin \left(\frac{\Omega t}{2}\right)\right)
\end{aligned}
$$

With $\omega^{2}=\frac{\Omega^{2}}{4}+\Omega \delta$ to the lowest order in $\delta$, we insert Eqs. (22) and (24) in Eq. (16) to write

$$
\begin{aligned}
& -\Omega \dot{A}(t) \sin \left(\frac{\Omega t}{2}\right)+\Omega \dot{B}(t) \cos \left(\frac{\Omega t}{2}\right) \\
& +\delta \epsilon \Omega\left(A(t) \cos \left(\frac{\Omega t}{2}\right)+B(t) \sin \left(\frac{\Omega t}{2}\right)\right) \\
& +\epsilon \cos (\Omega t)\left(A(t) \cos \left(\frac{\Omega t}{2}\right)\right. \\
& \left.+B(t) \sin \left(\frac{\Omega t}{2}\right)\right)=0
\end{aligned}
$$

Setting the coefficients of $\cos \left(\frac{\Omega t}{2}\right)$ and $\sin \left(\frac{\Omega t}{2}\right)$ to zero, we get

$$
\begin{aligned}
& \dot{A}=\left(\delta-\frac{\epsilon}{2 \Omega}\right) B \\
& \dot{B}=-\left(\delta+\frac{\epsilon}{2 \Omega}\right) A
\end{aligned}
$$

leading to the solution

$A(t)=C_{1} e^{i\left(\sqrt{\delta^{2}-\frac{\epsilon^{2}}{4 \Omega^{2}}}\right) t}+C_{2} e^{-i\left(\sqrt{\delta^{2}-\frac{\epsilon^{2}}{4 \Omega^{2}}}\right) t}$
The answer reveals that the slow variation of $A(t)$ comes from the fact the coefficient of $t$ in the exponential is small. It also shows that if $\delta^{2}>\frac{\epsilon^{2}}{4 \Omega^{2}}$, the solutions are periodic with frequency $\sqrt{\delta^{2}-\frac{\epsilon^{2}}{4 \Omega^{2}}}$ and hence the solution for $X(t)$ [as seen from Eq. (22)] is quasi-periodic. For $\delta= \pm \frac{\epsilon}{2 \Omega}$, the solution $X(t)$ is periodic as $A(t)$ and $B(t)$ become constants. This agree exactly with the curves we found from the perturbation theory for periodic solutions. For $\delta<\left|\frac{\epsilon}{2 \Omega}\right|$ (the region bounded by the curves where the solution is periodic), $X(t)$ diverges. Hence, we have a situation where quasi-periodic solutions are separated from diverging solutions by curves on which the solution is periodic. Searching for periodic solutions is a good strategy for finding zones where the solutions may diverge. It should be noted that the Krylov-Bogoliubov technique is extremely versatile as it gives a complete picture of what is going on, in the vicinity of the periodic solution. It should be noted that the multiple timescale technique (see Ref. [29]) yields identical answers. The numerically obtained picture of solutions of the Mathieu equation are shown in Fig. 1.

To solve the higher-order differential equations, we used the Runge-Kutta (RK) recursion formula. Higherorder differential equations can be represented by a set of first-order differential equations. Runge-Kutta algorithm has the form $y_{i+1}=y_{i}+h \phi\left(x_{i}, y_{i}, h\right)$, to numerically solve the first-order differential equation, where $\phi$ is an increment function of the approximation of

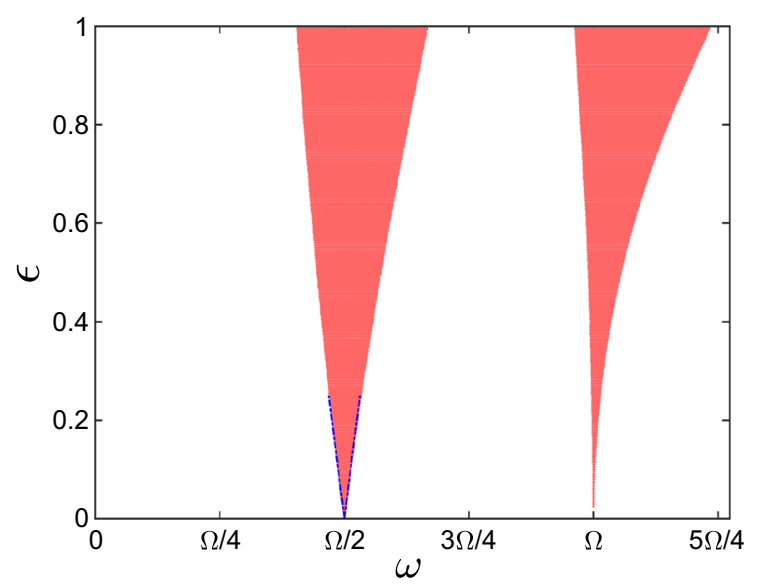

Fig. 1 Zones of bounded and unbounded solutions for $\langle x\rangle$. The red shaded regions show the unstable zones. The boundaries obtained around $\omega=\frac{\Omega}{2}$ from perturbation theory are shown by the blue dashed lines. (Color figure online) 
$f(x, y)$ in the interval of $x_{i} \leq x \leq x_{i+1}$. We have used fourth-order RK formulas, where $\phi$ is the weighted average of four derivative evaluations $k_{1}, k_{2}, k_{3}$ and $k_{4}$ on the interval $x_{i} \leq x \leq x_{i+1}$, that is,

$y_{i+1}=y_{i}+\frac{h}{6}\left(k_{1}+2 k_{2}+2 k_{3}+k_{4}\right)$,

where,

$k_{1}=f\left(x_{i}, y_{i}\right)$,

$k_{2}=f\left(x_{i}+\frac{h}{2}, y_{i}+\frac{h}{2} k_{1}\right)$,

$k_{3}=f\left(x_{i}+\frac{h}{2}, y_{i}+\frac{h}{2} k_{2}\right)$,

$k_{4}=f\left(x_{i}+h, y_{i}+h k_{3}\right)$.

We have used a step size of $h=0.01$. Smaller values of $h$ do not change the computed boundaries.

With this introduction to the techniques to be used, we now turn to a fairly elaborate discussion of the thirdorder equation Eq. (14) satisfied by $V(t)$. The derivation of the equation is given in "Appendix A".

\section{The properties of the third-order non-autonomous equation for the variance}

The general discussions of Eqs. (10-12) from the mathematical point of view can be found in Refs. [19-22]. We begin by noting the fact that Eq. (13) can have periodic motion with periods $3 T, 2 T$ and $T$ and by asking for the region in $(\bar{\omega}-\epsilon)$ space where the solution of period $3 T$ should be obtained. This has to be the primary resonance near $\bar{\omega}=\frac{\Omega}{3}$ and we write

$\bar{\omega}=\frac{\Omega}{3}+\Delta \bar{\omega}(\epsilon)=\frac{\Omega}{3}+\delta_{1} \epsilon+\delta_{2} \epsilon^{2}+\cdots$

Perturbation theory is what we want to begin with and write

$$
V=V_{0}+\epsilon V_{1}+\epsilon^{2} V_{2}+\cdots
$$

Substituting the expansion in Eq. (13), we have

$$
\begin{aligned}
& \left(\dddot{V}_{0}+\epsilon \dddot{V}_{1}+\epsilon^{2} \dddot{V}_{2}+. .\right)+\left(\frac{\Omega^{2}}{9}+2 \frac{\Omega}{3} \delta_{1} \epsilon\right. \\
& \left.+2 \frac{\Omega}{3} \delta_{2} \epsilon^{2}\right)\left(\dot{V}_{0}+\epsilon \dot{V}_{1}+\epsilon^{2} \dot{V}_{2}+. .\right)+\epsilon \cos (\Omega t) \\
& \left(\dot{V}_{0}+\epsilon \dot{V}_{1}+. .\right)-\frac{\epsilon \Omega}{2} \sin (\Omega t)\left(V_{0}+\epsilon V_{1}+. .\right)=0
\end{aligned}
$$

The analysis of the above equation up to $\mathcal{O}\left(\epsilon^{2}\right)$ is presented in "Appendix B". We find that there is no zone of instability to $\mathcal{O}\left(\epsilon^{2}\right)$ and the periodic orbits appear on the curve $\bar{\omega}=-\frac{27 \epsilon^{2}}{128 \Omega^{3}}$.

We now focus on the orbits of period $2 T$. A harmonic balance argument suggests that these will exist in the vicinity of $\bar{\omega}=\frac{\Omega}{2}$. We write $\bar{\omega}=\frac{\Omega}{2}+\delta$, where $\delta$ is small and try a Krylov-Bogoliubov technique with the trial solution

$$
\begin{aligned}
V(t)= & A_{1}(t) \cos \left(\frac{\Omega t}{2}\right)+B_{1}(t) \sin \left(\frac{\Omega t}{2}\right) \\
& +A_{2}(t) \cos \left(\frac{3 \Omega t}{2}\right)+B_{2}(t) \sin \left(\frac{3 \Omega t}{2}\right)
\end{aligned}
$$

We provide the expressions for $\dot{V}, \ddot{V}$ and $\dddot{V}$ in "Appendix B".

From the coefficients of $\sin \left(\frac{\Omega t}{2}\right), \cos \left(\frac{\Omega t}{2}\right), \sin \left(\frac{3 \Omega t}{2}\right)$ and $\cos \left(\frac{3 \Omega t}{2}\right)$ in Eq. (B.10) of "Appendix B",

$$
\begin{aligned}
& -\frac{\Omega^{2}}{2} \dot{A}_{1}+\frac{\delta \Omega^{2}}{2} B_{1}+\frac{\epsilon \Omega}{2} B_{2}=0 \\
& -\frac{\Omega^{2}}{2} \dot{B}_{1}-\frac{\delta \Omega^{2}}{2} A_{1}-\frac{\epsilon \Omega}{2} A_{2}=0 \\
& -\frac{13 \Omega^{2}}{2} \dot{A}_{2}-3 \Omega^{3} B_{2}+\frac{\epsilon \Omega}{2} B_{1}=0 \\
& -\frac{13 \Omega^{2}}{2} \dot{B}_{2}+3 \Omega^{3} A_{2}-\frac{\epsilon \Omega}{2} A_{1}=0
\end{aligned}
$$

The eigenvalues $\lambda$ of this set of equations are obtained from the condition

$$
\left|\begin{array}{cccc}
\lambda & -\delta & 0 & -\frac{\epsilon}{\Omega} \\
\delta & \lambda & \frac{\epsilon}{\Omega} & 0 \\
0 & -\frac{\epsilon}{13 \Omega} & \lambda & \frac{6 \Omega}{13} \\
\frac{13 \epsilon}{\Omega} & 0 & -\frac{6 \Omega}{13} & \lambda
\end{array}\right|=0
$$

which gives the following quadratic equation in $\lambda^{2}$ :

$$
\begin{gathered}
\lambda^{4}+\lambda^{2}\left(\frac{36 \Omega^{2}}{169}+\delta^{2}\right)+\frac{36 \Omega^{2} \delta^{2}}{169} \\
+\frac{12 \epsilon^{2} \delta}{169 \Omega}+\frac{\epsilon^{4}}{169 \Omega^{4}}=0
\end{gathered}
$$

The two roots for $\lambda^{2}$ are obtained as

$$
\begin{aligned}
\lambda^{2}= & -\frac{1}{2}\left(\frac{36 \Omega^{2}}{169}+\delta^{2}\right) \\
& \pm \sqrt{\frac{1}{4}\left(\frac{36 \Omega^{2}}{169}+\delta^{2}\right)^{2}-\left(\frac{36 \Omega^{2} \delta^{2}}{169}+\frac{12 \epsilon^{2} \delta}{169 \Omega}+\frac{\epsilon^{4}}{169 \Omega^{4}}\right)}
\end{aligned}
$$


We note that neither of the roots can be positive, and hence, there is no instability zone around $\bar{\omega}=$ $\frac{\Omega}{2}$. The periodic orbit is obtained for $\lambda=0$ which corresponds to $\delta=-\frac{\epsilon^{2}}{6 \Omega^{3}}$. This is interesting since the anticipated instability zone is not there. It can be traced to an accidental cancellation of terms in $\cos \left(\frac{\Omega t}{2}\right)$ and $\sin \left(\frac{\Omega t}{2}\right)$ coming from the third and fourth terms of Eq. (14).

We now try to find out the solutions of period $T$ which are expected to occur near $\bar{\omega}=\Omega$. Setting $\bar{\omega}=$ $\Omega+\delta$, we carry out the Krylov-Bogoliubov method with the ansatz,

$$
\begin{aligned}
V(t)= & A_{0}(t)+A_{1}(t) \cos (\Omega t)+B_{1}(t) \sin (\Omega t) \\
& +A_{2}(t) \cos (2 \Omega t)+B_{2}(t) \sin (2 \Omega t)
\end{aligned}
$$

where $A_{0}, A_{1}, B_{1}, A_{2}, B_{2}$ are all slowly varying function of time. Our calculation technique is exactly the same as detailed in the derivation of Eqs. (29)-(32d) and finally yields

$$
\begin{aligned}
& \dot{A}_{0}=-\frac{\epsilon \Omega}{4} B_{1} \\
& \dot{A}_{1}=\frac{2 \delta}{\Omega} B_{1}+\frac{3}{8} \epsilon \Omega B_{2} \\
& \dot{B}_{1}=-\frac{2 \delta}{\Omega} A_{1}-\frac{3}{8} \epsilon \Omega A_{2}-\frac{\epsilon \Omega}{4} A_{0} \\
& \dot{A}_{2}=-\frac{6}{11} \Omega B_{2} \\
& \dot{B}_{2}=\frac{6}{11} \Omega A_{2}
\end{aligned}
$$

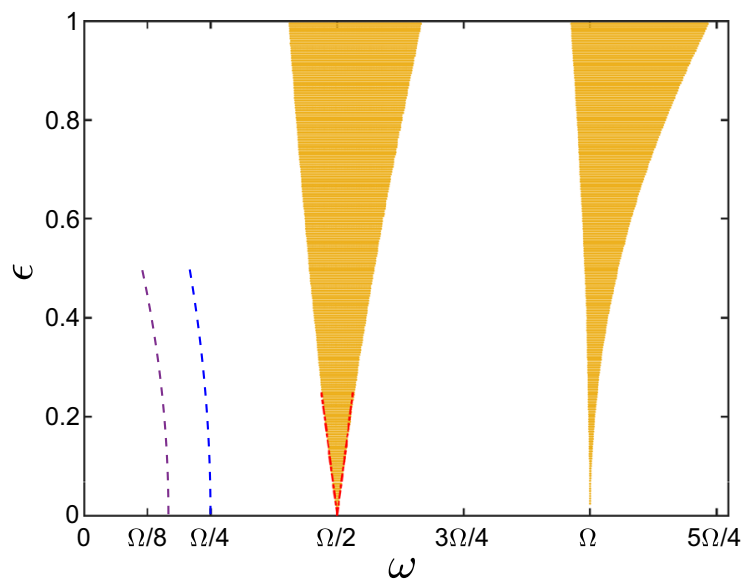

Fig. 2 Zones of bounded and unbounded solutions for $\Delta^{2}$. The golden colour shaded regions show the unstable zones. The dashed line shows where periodic orbits occur according to perturbation theory. The difference from Fig. 1 lies in the extra periodic line originating at $\omega=\frac{\Omega}{4}$. (Color figure online)
The eigenvalue $\lambda$ satisfies a fifth-order equation which always has a zero eigenvalue. Of the four remaining eigenvalues, two are purely imaginary and the other two have the form $\pm \sqrt{\frac{\epsilon^{2} \Omega^{2}}{16}-4 \delta^{2}}$, and hence, there is a zone of divergent solution bounded by $\delta= \pm \frac{\epsilon}{8}$ exactly as for the Mathieu equation near $\omega=\frac{\Omega}{2}$.

We have solved Eq. (14) numerically using RungeKutta methods to obtained the instability zones as shown in Fig. 2. The instability zones agree exactly with those found in Fig. 1 for small values of $\epsilon$. As seen from our calculation, the first instability zone appears at $\bar{\omega}=2 \omega=\Omega$ and its width is exactly the same as the instability zone in Fig. 1 around $\omega=\frac{\Omega}{2}$. This explains why the recent numerical work of Hashemloo et al. [27] finds that the width increases indefinitely only in the parameters zones where the mean position increases. We end this section by noting that the absence of an instability zone around $\bar{\omega}=\frac{\Omega}{3}$ (which corresponds to $\omega=\frac{\Omega}{6}$ ) is presumably due to the fact that in the absence of the periodic forcing, the system exhibits a zero mode. The absence of a zone around $\omega=\frac{\Omega}{4}$ is accidental because of the coefficients of $f$ and $\dot{f}$ in Eq. (10) differing exactly by a factor of two.

\section{The properties of the fourth- and fifth-order equations for the skewness and kurtosis}

We begin our discussion with the skewness $S$, rewriting Eq. (11) to $\mathcal{O}(\epsilon)$. This leads to

$$
\begin{aligned}
& \frac{\mathrm{d}^{4}}{\mathrm{~d} t^{4}} S+10 \omega^{2} \frac{\mathrm{d}^{2}}{\mathrm{~d} t^{2}} S+9 \omega^{4} S+10 \omega^{2} \epsilon f(t) \frac{\mathrm{d}^{2}}{\mathrm{~d} t^{2}} S \\
& +18 \omega^{4} \epsilon f(t) S+10 \epsilon \omega^{2} \dot{f} \frac{\mathrm{d}}{\mathrm{d} t} S+3 \omega^{2} \epsilon \ddot{f} S=0
\end{aligned}
$$

with $f=\cos (\Omega t)$. Writing this as a fourth-order dynamical system $\dot{X}_{i}=A_{i j} X_{j}$ with periodic coefficients, we find that $M_{i j}$ is traceless and hence the solutions $X(t)$ can have periodicities $4 T, 3 T, 2 T$ and $T$. There are solutions with frequency $\frac{\Omega}{4}, \frac{\Omega}{3}, \frac{\Omega}{2}$ and $\Omega$. The difference between Eq. (37) and the system studied in Sects. 2 and 3 is that the unperturbed solution $S_{0}$ of Eq. (37) [i.e. solution for $\epsilon=0$ ] is a two-frequency solution (this is a significant difference that can occur with the higher-order Mathieu equation)

$$
\begin{gathered}
S_{0}(t)=A_{1} \cos (\omega t)+B_{1} \sin (\omega t)+A_{2} \cos (3 \omega t) \\
+B_{2} \sin (3 \omega t) .
\end{gathered}
$$




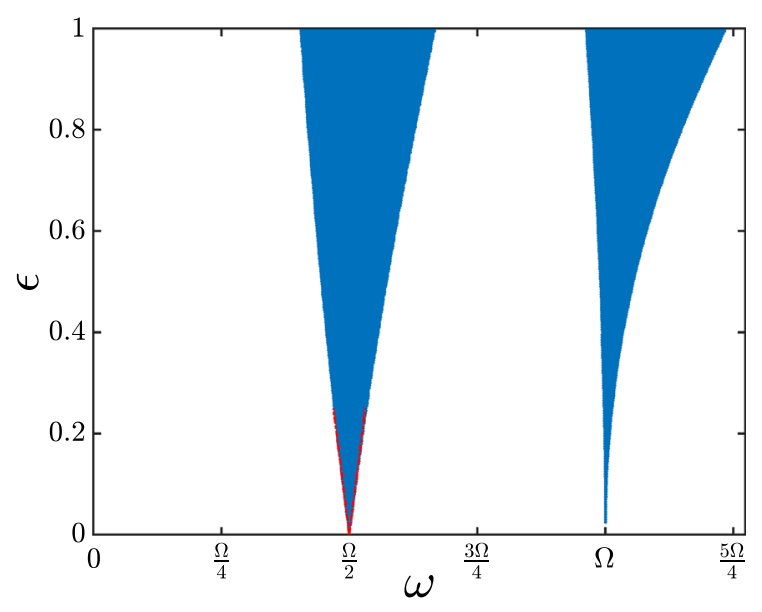

Fig. 3 Zones of bounded and unbounded solutions for skewness. The blue shaded regions show the unstable zones. The red dashed lines show the boundaries obtained from perturbation theory. (Color figure online)

The expected resonant response can happen when either $\omega$ or $3 \omega$ matches $\frac{\Omega}{4}, \frac{\Omega}{3}, \frac{\Omega}{2}$ and $\Omega$. Harmonic balance indicates which matching would lead to dominant effect and inspection shows that the conditions $\omega=\frac{\Omega}{6}$ or $\frac{\Omega}{2}$ are the ones capable of producing response at $\mathcal{O}(\epsilon)$. The details of the calculations are presented in "Appendix C". Here we simply report that there is no divergent region near $\omega=\frac{\Omega}{6}$, while in the vicinity of $\omega=\frac{\Omega}{2}$, we have the boundaries given by the curves shown in Eq. (C.8a).

We show the instability zones obtained from our computational work and the boundaries obtained from perturbation theory in Fig. 3.

We now turn to the dynamics of the kurtosis $K$ Eq. (12) and writing it as a dynamical system find that once again the matrix $A$ is traceless and the possible time periods of the solution can be $5 T, 4 T, \ldots, T$ with frequencies $\frac{\Omega}{5}, \frac{\Omega}{4}, \ldots, \Omega$. Noting that the $\epsilon=0$ system has a solution with frequencies $2 \omega$ and $4 \omega$, we find from a visual inspection of harmonic balance that at $\mathcal{O}(\epsilon)$, the possibilities are the matches at $\omega=\frac{\Omega}{8}$ and $\omega=\frac{\Omega}{4}$ and $\omega=\frac{\Omega}{2}$. We rewrite Eq. (12) in a more streamlined form by writing $2 \omega$ as $\bar{\omega}$ and $\epsilon \bar{\omega}^{2}$ as $\bar{\epsilon}$. With there new redefinition, we have (keeping terms to $\mathcal{O}(\bar{\epsilon})$ only as our calculations will be done to the order)

$$
\begin{gathered}
\dddot{K}+5 \bar{\omega}^{2} \dddot{K}+4 \bar{\omega}^{4} \dot{K}=-5 \bar{\epsilon} \cos (\Omega t) \dddot{K} \\
-8 \bar{\omega}^{2} \bar{\epsilon} \cos (\Omega t) \dot{K}+6 \bar{\epsilon} \Omega \sin (\Omega t) \ddot{K}
\end{gathered}
$$

$$
\begin{aligned}
& +\frac{9}{2} \bar{\epsilon} \Omega^{2} \cos (\Omega t) \dot{K}+4 \bar{\omega}^{2} \bar{\epsilon} \Omega \sin (\Omega t) K \\
& -\Omega^{3} \bar{\epsilon} \sin (\Omega t) K
\end{aligned}
$$

To explore $\omega$ near $\frac{\Omega}{8}$, we need to set $\bar{\omega}=\frac{\Omega}{4}+\delta$. Expanding $\delta$ as $\delta_{1} \bar{\epsilon}+\delta_{2} \bar{\epsilon}^{2}$, we retain the first term alone and write Eq. (39) as

$$
\begin{aligned}
\dddot{K}+ & 5 \frac{\Omega^{2}}{16} \dddot{K}+4\left(\frac{\Omega}{4}\right)^{4} \dot{K}+\frac{5 \Omega}{2} \delta_{1} \bar{\epsilon} \dddot{K}+\frac{\Omega^{3}}{4} \delta_{1} \bar{\epsilon} \dot{K} \\
= & -5 \bar{\epsilon} \cos (\Omega t) \dddot{K}-\frac{\Omega^{2}}{2} \bar{\epsilon} \cos (\Omega t) \dot{K} \\
& +6 \bar{\epsilon} \Omega \sin (\Omega t) \ddot{K} \\
& +\frac{9}{2} \bar{\epsilon} \Omega^{2} \cos (\Omega t) \dot{K}-\frac{3}{4} \bar{\epsilon} \Omega^{3} \sin (\Omega t) K
\end{aligned}
$$

At $\mathcal{O}(1)$

$\dddot{K}_{0}+5 \frac{\Omega^{2}}{16} \dddot{K}_{0}+4\left(\frac{\Omega}{4}\right)^{4} \dot{K}_{0}=0$

with the solution

$$
\begin{aligned}
K_{0}= & A_{0}+A_{1} \cos \left(\frac{\Omega}{4} t\right)+B_{1} \sin \left(\frac{\Omega}{4} t\right) \\
& +A_{2} \cos \left(\frac{\Omega}{2} t\right)+B_{2} \sin \left(\frac{\Omega}{2} t\right)
\end{aligned}
$$

At $\mathcal{O}(\bar{\epsilon})$

$$
\begin{aligned}
\dddot{K}_{1} & +5 \frac{\Omega^{2}}{16} \dddot{K}_{1}+4\left(\frac{\Omega}{4}\right)^{4} \dot{K}=-\frac{5 \Omega}{2} \delta_{1} \dddot{K}_{0} \\
& -\frac{\Omega^{3}}{4} \delta_{1} \dot{K}_{0}-5 \cos (\Omega t) \dddot{K}_{0}+4 \Omega^{2} \cos (\Omega t) \dot{K}_{0} \\
& +6 \Omega \sin (\Omega t) \ddot{K}_{0}-\frac{3}{4} \Omega^{3} \sin (\Omega t) K_{0}
\end{aligned}
$$

The relevant resonance-inducing terms on the r.h.s. of Eq. (41) are of the two varieties: one set proportional to $A_{1} \cos \left(\frac{\Omega}{4}\right)$ and $B_{1} \sin \left(\frac{\Omega}{4}\right)$ which have $\delta_{1}$ as a prefactor and the other set proportional to $A_{2} \cos \left(\frac{\Omega}{2}\right)$ and $B_{2} \sin \left(\frac{\Omega}{2}\right)$ where coefficients contain terms with $\delta_{1}$ and also without. Setting $\delta_{1}=0$ to remove resonance is not an option. Hence, the resonance is removed by setting $A_{1}=B_{1}=0$ and

$$
\begin{aligned}
\delta_{1} & =+\frac{1}{\Omega} \quad \text { for the solution } A_{2} \cos \left(\frac{\Omega t}{2}\right) \\
& =-\frac{1}{\Omega} \quad \text { for the solution } B_{2} \sin \left(\frac{\Omega t}{2}\right)
\end{aligned}
$$

This produces a zone starting at $\omega=\frac{\Omega}{8}$ where the solutions diverge in time. On the boundaries of this zone, the solution is periodic with a period $2 T$. 
An identical calculation holds near $\omega=\frac{\Omega}{4}$, but now the width of the zone is exactly double that the zone around $\omega=\frac{\Omega}{8}$.

We finally consider the case $\omega=\frac{\Omega}{2}+\delta$. In this case, as for $V(t)$, the perturbative technique used above does not work. With $\omega=\frac{\Omega}{2}+\delta$, we write Eq. (12) to the lowest order in $\delta$ and $\epsilon$ as

$$
\begin{aligned}
\frac{\mathrm{d}^{5} K}{\mathrm{~d} t^{5}} & +5 \Omega^{2} \frac{\mathrm{d}^{3} K}{\mathrm{~d} t^{3}}+4 \Omega^{4} \frac{\mathrm{d} K}{\mathrm{~d} t} \\
= & -20 \Omega \delta \frac{\mathrm{d}^{3} K}{\mathrm{~d} t^{3}}-32 \Omega^{3} \delta \frac{\mathrm{d} K}{\mathrm{~d} t} \\
& -5 \epsilon \Omega^{2} \cos (\Omega t) \frac{\mathrm{d}^{3} K}{\mathrm{~d} t^{3}}+6 \epsilon \Omega^{3} \sin (\Omega t) \frac{\mathrm{d}^{2} K}{\mathrm{~d} t^{2}} \\
& -\frac{7}{2} \epsilon \Omega^{4} \cos (\Omega t) \frac{\mathrm{d} K}{\mathrm{~d} t}+3 \epsilon \Omega^{5} \sin (\Omega t) K
\end{aligned}
$$

We use the Krylov-Bogoliubov method with

$$
\begin{aligned}
K(t)= & A_{0}(t)+A_{1}(t) \cos (\omega t)+B_{1}(t) \sin (\omega t) \\
& +A_{2}(t) \cos (2 \omega t)+B_{2}(t) \sin (2 \omega t) \\
& +A_{3}(t) \cos (3 \omega t)+B_{3}(t) \sin (3 \omega t)
\end{aligned}
$$

where $A_{0}, A_{1}, A_{2}, A_{3}, B_{1}, B_{2}, B_{3}$ are slowly varying function of time. We had to include three harmonics in this case as opposed to the two harmonics in Eqs. (31) and (35) because the zeroth-order solution of Eq. (44) has the structure $K=A_{0}+A_{1} \cos (\omega t)+B_{1} \sin (\omega t)+$ $A_{2} \cos (2 \omega t)+B_{2} \sin (2 \omega t)$. At $\mathcal{O}(\epsilon)$, we consequently generate the $\cos (3 \omega t)$ and $\sin (3 \omega t)$ terms which need to be in the Krylov-Bogoliubov method.

Straightforward algebra now leads to

$$
\begin{aligned}
& \dot{A}_{0}=-\frac{3 \epsilon}{16} B_{1} \\
& \dot{A}_{1}=2 \delta B_{1}-\epsilon B_{2} \\
& \dot{B}_{1}=-2 \delta A_{1}+\epsilon A_{2}-\frac{\epsilon}{2} A_{0} \\
& \dot{A}_{2}=4 \delta B_{2}+\frac{3 \epsilon}{32} B_{1}+\frac{49}{32} \epsilon B_{3} \\
& \dot{B}_{2}=-4 \delta A_{2}-\frac{3 \epsilon}{32} A_{1}-\frac{49}{32} \epsilon A_{3} \\
& \dot{A}_{3}=-\frac{60}{137} B_{3}+\frac{27}{274} \epsilon B_{2} \\
& \dot{B}_{3}=\frac{60}{137} A_{3}-\frac{27}{274} \epsilon A_{2}
\end{aligned}
$$

As expected there is a zero mode, as there was for the variance. The other eigenvalues are $\lambda= \pm i \frac{60}{137}+\mathcal{O}(\epsilon)$ and the remaining four obtained from the solution of $\lambda^{4}+\lambda^{2}\left(20 \delta^{2}-\frac{9 \epsilon^{2}}{32}\right)+64 \delta^{4}+\frac{153}{1024} \epsilon^{4}=0$

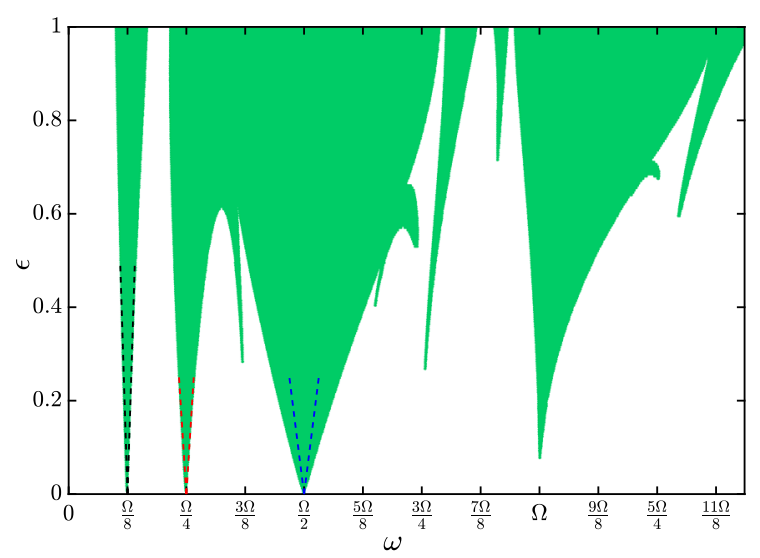

Fig. 4 Zones of bounded and unbounded solutions for kurtosis. The green shaded regions show the unstable zones. The dashed lines show the calculated instability zones. For $\omega=\frac{\Omega}{2}$, it is discussed why the actual bounding can be somewhat wider. (Color figure online)

For real value of $\lambda^{2}$, one requires $\left(20 \delta^{2}-\frac{9 \epsilon^{2}}{32}\right)>$ $256 \delta^{4}+\frac{153}{256} \epsilon^{4}$. If $\lambda^{2}$ is real, then clearly for $\lambda^{2}$ to have a positive root(instability) $20 \delta^{2}<\frac{9 \epsilon^{2}}{32}$ which would mean $\delta^{2}<\frac{9 \epsilon^{2}}{640}$ which would imply the unstable zone is $\delta<\left|\frac{3}{8 \sqrt{10}}\right| \epsilon$ which is very close to $\frac{\epsilon}{8}$. However, this is not the complete story since this is true only when $\lambda^{2}$ has real roots. If $\lambda$ has complex roots with $\lambda^{2}$ having the structure $\lambda^{2}=-\alpha \pm i \beta$, where $\alpha$ is positive being in the stable zone $\delta>\left|\frac{3}{8 \sqrt{10}}\right| \epsilon$ then $\lambda=\sqrt[4]{\alpha^{2}+\beta^{2}} e^{ \pm i \theta / 2}$ where $\cos (\theta)=\frac{\alpha}{\sqrt{\alpha^{2}+\beta^{2}}}$ and $\sin (\theta)=\frac{\beta}{\sqrt{\alpha^{2}+\beta^{2}}}$. A negative $\cos (\theta)$ puts $\theta$ in the range $\frac{\pi}{2}$ to $\frac{3 \pi}{2}$ and $\cos \left(\frac{\theta}{2}\right)$ is in the range $\frac{\pi}{4}$ to $\frac{3 \pi}{4}$. This implies $\cos \left(\frac{\theta}{2}\right)$ is positive for $\frac{\pi}{2}<\theta<\frac{3 \pi}{4}$ and this is what magnifies the instability zone around $\omega=\frac{\Omega}{2}$, way beyond what happens for $\langle x\rangle, V$ and $S$. As can be seen from Fig. 4, our perturbative calculations are in accordance with the numerical data. It should be seen that at this order of Mathieu equation, the main feature of instabilities arising at lower frequencies than the second-order equation is clearly borne out as the accidental cancellation no longer happen. However, in this case as $\epsilon$ increases, the region in parameter space where the kurtosis diverges becomes very large as seen from our numerical work and this makes the long time quantum dynamics in such systems of particular interest. The long time shape of the probability distribution needs to have a power law fall off in the regions where the kurtosis diverges. 


\section{Conclusions}

Linear differential equations with periodic coefficients and of order greater than two are a rarity physical sciences. The quantum parametric oscillator provides a real physical system [24-27] where such equations make a natural appearance when the dynamics of higher moments is considered. Since description of the quantum state is in terms of probabilities one studies the dynamics of the mean value $\langle x\rangle$ instead of the position of $x$ itself. But the mean is not sufficient to picture the distribution and hence moments like the variance are essential. The fact that the moments follow Mathieu equations of higher order imply that while the mean may remain bounded, the fluctuation around the mean or the spread parameter kurtosis may diverge with time leading a drastic change in shape of the probability distribution. Hence, the quantum parametric oscillator provides the physical justification for investigating higher-order Mathieu equations.

The discussion on higher-order Mathieu-like equations have till now been restricted to mathematical analysis. We wanted to explore the practical issue of whether the perturbation techniques that work so well for finding the instability zones of the Mathieu equations would be equally useful for higher-order Mathieu equation. As seen from Figs. 2, 3 and 4, that is indeed the case. It will be noted that we never found any instability zone around lowest admissible frequency $\omega=\frac{\Omega}{6}$ in our study of the variance in Sect. 3. This we attribute to the existence of a zero mode in the system. The fact that there was no instability zone around $\omega=\frac{\Omega}{4}$ was due to an accidental cancellation. This did not occur for the kurtosis and instability zones can be seen emanating from $\omega=\frac{\Omega}{4}$ and $\omega=\frac{\Omega}{8}$ in Fig. 4. From a purely technical point of view, the fact that traditional technique like harmonic balance and Krylov-Bogoliubov provides results which could be verified in their zones of validity by numerical analysis indicates the robustness of these methods and should lead to interesting future investigations.

\section{Compliance with ethical standards}

Conflict of interest The authors declared no potential conflicts of interest with respect to the research, authorship of this article.

Open Access This article is distributed under the terms of the Creative Commons Attribution 4.0 International License (http:// creativecommons.org/licenses/by/4.0/), which permits unrestricted use, distribution, and reproduction in any medium, provided you give appropriate credit to the original author(s) and the source, provide a link to the Creative Commons license, and indicate if changes were made.

\section{Appendix A}

In the appendix, we provide the derivation of the dynamics of the variance $V=\left\langle x^{2}\right\rangle-\langle x\rangle^{2}$ which is the first signature of the existence of genuine quantum effects. Using the Ehrenfest equation shown in Eq. (1) with $\mathcal{O}=x^{2}$, we get

$$
\begin{aligned}
& \frac{\mathrm{d}}{\mathrm{d} t}\left\langle x^{2}\right\rangle=\frac{\langle x p+p x\rangle}{m} \\
& \frac{\mathrm{d}^{2}}{\mathrm{~d} t^{2}}\left\langle x^{2}\right\rangle=\frac{1}{m} \frac{\mathrm{d}}{\mathrm{d} t}\langle x p+p x\rangle \\
& \quad=2 \frac{\left\langle p^{2}\right\rangle}{m^{2}}+2 \omega^{2}\left\langle x^{2}\right\rangle(1+\epsilon f(t))
\end{aligned}
$$

At this point, we need to point out that if an operator $\mathcal{O}$ has explicit time dependence, then Eq. (1) becomes $i \hbar \frac{\mathrm{d}}{\mathrm{d} t}\langle\mathcal{O}\rangle=i \hbar \frac{\partial}{\partial t}\langle\mathcal{O}\rangle+\langle[\mathcal{O}, \mathcal{H}]\rangle$

Another derivative of Eq. (A.2) yields

$$
\begin{aligned}
\frac{\mathrm{d}^{3}}{\mathrm{~d} t^{3}}\left\langle x^{2}\right\rangle= & \frac{2}{m^{2}} \frac{\mathrm{d}}{\mathrm{d} t}\left\langle p^{2}\right\rangle-2 \omega^{2}(1+\epsilon f(t)) \frac{\mathrm{d}}{\mathrm{d} t}\left\langle x^{2}\right\rangle \\
& -2 \omega^{2} \epsilon \dot{f}\left\langle x^{2}\right\rangle
\end{aligned}
$$

Using Eq. (1) with $\mathcal{O}=p^{2}$ gives

$$
\begin{aligned}
\frac{\mathrm{d}}{\mathrm{d} t}\left\langle p^{2}\right\rangle & =-m \omega^{2}(1+\epsilon f(t))\langle x p+p x\rangle \\
& =-m^{2} \omega^{2}(1+\epsilon f(t)) \frac{\mathrm{d}}{\mathrm{d} t}\left\langle x^{2}\right\rangle
\end{aligned}
$$

where in the second step Eq. (A.1) is used. Substituting the above in Eq. (A.4) yields

$$
\frac{\mathrm{d}^{3}}{\mathrm{~d} t^{3}}\left\langle x^{2}\right\rangle=-4 \omega^{2}(1+\epsilon f(t)) \frac{\mathrm{d}}{\mathrm{d} t}\left\langle x^{2}\right\rangle-2 \omega^{2} \epsilon \dot{f}\left\langle x^{2}\right\rangle
$$

The expression for the $\frac{\mathrm{d}^{3}}{\mathrm{~d} t^{3}}\langle x\rangle^{2}$ can be found from Eqs. (3) and (4) and subtracting that from Eq. (6), we obtain Eq. (10).

\section{Appendix B}

In this appendix, we provide the details behind the perturbation analysis of the dynamics of the variance present in Sect. 3. We begin with Eq. (30) and note that at $\mathcal{O}(1)$, we get 
$\dddot{V}_{0}+\frac{\Omega^{2}}{9} \dot{V}_{0}=0$

with

$$
\begin{gathered}
V_{0}=A_{0}+A_{1} \cos \left(\frac{\Omega}{3} t\right)+B_{1} \sin \left(\frac{\Omega}{3} t\right) \\
\dddot{V}_{1}+\frac{\Omega^{2}}{9} \dot{V}_{1}+2 \frac{\Omega}{3} \delta_{1} \dot{V}_{0}+\cos (\Omega t) \dot{V}_{0} \\
-\frac{\Omega}{2} \sin (\Omega t) V_{0}=0
\end{gathered}
$$

Using Eq. (B.2)

$$
\begin{aligned}
\dddot{V}_{1} & +\frac{\Omega^{2}}{9} \dot{V}_{1} \\
= & -2 \frac{\Omega}{3} \delta_{1}\left(-A_{1} \frac{\Omega}{3} \sin \left(\frac{\Omega}{3} t\right)+B_{1} \frac{\Omega}{3} \cos \left(\frac{\Omega}{3} t\right)\right) \\
& -\cos (\Omega t)\left(-A_{1} \frac{\Omega}{3} \sin \left(\frac{\Omega}{3} t\right)+B_{1} \frac{\Omega}{3} \cos \left(\frac{\Omega}{3} t\right)\right) \\
& +\frac{\Omega}{2} \sin (\Omega t)\left(A_{0}+A_{1} \cos \left(\frac{\Omega}{3} t\right)+B_{1} \sin \left(\frac{\Omega}{3} t\right)\right) \\
= & -2 \frac{\Omega^{2}}{9} \delta_{1}\left(-A_{1} \sin \left(\frac{\Omega}{3} t\right)+B_{1} \cos \left(\frac{\Omega}{3} t\right)\right) \\
& +\frac{\Omega}{2} A_{0} \sin (\Omega t) \\
& +\Omega A_{1}\left(\frac{5}{12} \sin \left(\frac{4 \Omega t}{3}\right)+\frac{1}{12} \sin \left(\frac{2 \Omega t}{3}\right)\right) \\
& +\Omega B_{1}\left(\frac{1}{12} \cos \left(\frac{2 \Omega t}{3}\right)-\frac{5}{12} \cos \left(\frac{4 \Omega t}{3}\right)\right)
\end{aligned}
$$

For the solution $V_{1}$ to exist, we cannot have resonating terms on the right-hand side and hence $\delta_{1}=0$. At this order

$$
\begin{aligned}
V_{1}= & \frac{9}{16 \Omega} A_{0} \cos (\Omega t)+\frac{3}{16 \Omega^{2}} A_{1} \cos \left(\frac{4 \Omega t}{3}\right) \\
& +\frac{3}{8 \Omega^{2}} A_{1} \cos \left(\frac{2 \Omega t}{3}\right)-\frac{3}{8 \Omega^{2}} B_{1} \sin \left(\frac{2 \Omega t}{3}\right) \\
& +\frac{3}{16 \Omega^{2}} B_{1} \sin \left(\frac{4 \Omega t}{3}\right)
\end{aligned}
$$

$$
\begin{aligned}
& \text { At } \mathcal{O}\left(\epsilon^{2}\right) \\
& \dddot{V}_{2}+\frac{\Omega^{2}}{9} \dot{V}_{2} \\
& =-2 \frac{\Omega}{3} \delta_{2} \dot{V}_{0}-\cos (\Omega t) \dot{V}_{1}+\frac{\Omega}{2} \sin (\Omega t) V_{1} \\
& =-2 \frac{\Omega^{2}}{9} \delta_{2}\left(-A_{1} \sin \left(\frac{\Omega}{3} t\right)+B_{1} \cos \left(\frac{\Omega}{3} t\right)\right)
\end{aligned}
$$

$$
\begin{aligned}
& -\cos (\Omega t)\left[-\frac{9}{16} A_{0} \sin (\Omega t)-\frac{A_{1}}{4 \Omega} \sin \left(\frac{4 \Omega t}{3}\right)\right. \\
& -\frac{A_{1}}{4 \Omega} \sin \left(\frac{2 \Omega t}{3}\right)-\frac{B_{1}}{4 \Omega} \cos \left(\frac{2 \Omega t}{3}\right) \\
& \left.+\frac{B_{1}}{4 \Omega} \cos \left(\frac{4 \Omega t}{3}\right)\right]+\frac{\Omega}{2} \sin (\Omega t) \\
& {\left[\frac{9}{16 \Omega} A_{0} \cos (\Omega t)+\frac{3}{16 \Omega^{2}} A_{1} \cos \left(\frac{4 \Omega t}{3}\right)\right.} \\
& +\frac{3}{8 \Omega^{2}} A_{1} \cos \left(\frac{2 \Omega t}{3}\right)-\frac{3}{8 \Omega^{2}} B_{1} \sin \left(\frac{2 \Omega t}{3}\right) \\
& \left.+\frac{3}{16 \Omega^{2}} B_{1} \sin \left(\frac{4 \Omega t}{3}\right)\right]
\end{aligned}
$$

We need to pick out the resonance inducing secular terms from the right-hand side of Eq. (B.6) and set them equal to zero. This gives

$$
\begin{gathered}
\frac{2 \Omega^{2}}{9} \delta_{2} A_{1} \sin \left(\frac{\Omega t}{3}\right)-\frac{2 \Omega^{2}}{9} \delta_{2} B_{1} \cos \left(\frac{\Omega t}{3}\right) \\
\frac{3 A_{1}}{64 \Omega} \sin \left(\frac{\Omega t}{3}\right)-\frac{3 B_{1}}{64 \Omega} \cos \left(\frac{\Omega t}{3}\right)=0
\end{gathered}
$$

and hence we obtain $\delta_{2}=-\frac{27}{128 \Omega^{3}}$ for both $A_{1}=$ $0, B_{1} \neq 0$ and $A_{1} \neq 0, B_{1}=0$.

This indicates that the period $3 T$ orbit can exist only along the line $\bar{\omega}=-\frac{27}{128 \Omega^{3}} \epsilon^{2}+\mathcal{O}\left(\epsilon^{3}\right)$ in $\delta-\epsilon$ plane and there are no instability zone.

We now turn to Eq. (31) of the text which is the input for the Krylov-Bogoliubov technique for study of Eq. (14) near the frequency $\bar{\omega}=\frac{\Omega}{2}$. We consequently need the expression for $\dot{V}(t), \ddot{V}(t)$ and $\dddot{V}(t)$ keeping in mind that $A_{1}(t), B_{1}(t), A_{2}(t)$ and $B_{2}(t)$ of Eq. (31) are slowly varying quantities, hence the above mentioned derivatives of $V(t)$ will not contain any time derivatives of $A_{1}, B_{1}, A_{2}$ and $B_{2}$ higher than the second. With this, we get

$$
\begin{aligned}
\dot{V}(t)= & \dot{A}_{1}(t) \cos \left(\frac{\Omega t}{2}\right)+\dot{B}_{1}(t) \sin \left(\frac{\Omega t}{2}\right) \\
& +\dot{A}_{2}(t) \cos \left(\frac{3 \Omega t}{2}\right)+\dot{B}_{2}(t) \sin \left(\frac{3 \Omega t}{2}\right) \\
& +\frac{\Omega}{2}\left[-A_{1}(t) \sin \left(\frac{\Omega t}{2}\right)+B_{1}(t) \cos \left(\frac{\Omega t}{2}\right)\right] \\
& +\frac{3 \Omega}{2}\left[-A_{2}(t) \sin \left(\frac{3 \Omega t}{2}\right)+B_{2}(t) \cos \left(\frac{3 \Omega t}{2}\right)\right] \\
\ddot{V}(t)= & \Omega\left[-\dot{A}_{1}(t) \sin \left(\frac{\Omega t}{2}\right)+\dot{B}_{1}(t) \cos \left(\frac{\Omega t}{2}\right)\right] \\
& 3 \Omega\left[-\dot{A}_{2}(t) \sin \left(\frac{3 \Omega t}{2}\right)+\dot{B}_{2}(t) \cos \left(\frac{3 \Omega t}{2}\right)\right]
\end{aligned}
$$




$$
\begin{aligned}
& -\frac{\Omega^{2}}{4}\left[A_{1}(t) \cos \left(\frac{\Omega t}{2}\right)+B_{1}(t) \sin \left(\frac{\Omega t}{2}\right)\right] \\
& -\frac{9 \Omega^{2}}{4}\left[A_{2}(t) \cos \left(\frac{3 \Omega t}{2}\right)+B_{2}(t) \sin \left(\frac{3 \Omega t}{2}\right)\right]
\end{aligned}
$$

$$
\begin{aligned}
\dddot{V}(t)= & -\frac{3 \Omega^{2}}{4}\left[\dot{A}_{1}(t) \cos \left(\frac{\Omega t}{2}\right)+\dot{B}_{1}(t) \sin \left(\frac{\Omega t}{2}\right)\right] \\
& -\frac{27 \Omega^{2}}{4}\left[\dot{A}_{2}(t) \cos \left(\frac{3 \Omega t}{2}\right)+\dot{B}_{2}(t) \sin \left(\frac{3 \Omega t}{2}\right)\right] \\
& -\frac{\Omega^{3}}{8}\left[-A_{1}(t) \sin \left(\frac{\Omega t}{2}\right)+B_{1}(t) \cos \left(\frac{\Omega t}{2}\right)\right] \\
& -\frac{27 \Omega^{3}}{8}\left[-A_{2}(t) \sin \left(\frac{3 \Omega t}{2}\right)+B_{2}(t) \cos \left(\frac{3 \Omega t}{2}\right)\right]
\end{aligned}
$$

Inserting in Eq. (14) and keeping only terms in $\sin \left(\frac{\Omega t}{2}\right)$, $\cos \left(\frac{\Omega t}{2}\right), \sin \left(\frac{3 \Omega t}{2}\right)$ and $\cos \left(\frac{3 \Omega t}{2}\right)$, we have

$$
\begin{aligned}
- & \frac{3 \Omega^{2}}{4}\left[\dot{A}_{1}(t) \cos \left(\frac{\Omega t}{2}\right)+\dot{B}_{1}(t) \sin \left(\frac{\Omega t}{2}\right)\right] \\
- & \frac{27 \Omega^{2}}{4}\left[\dot{A}_{2}(t) \cos \left(\frac{3 \Omega t}{2}\right)+\dot{B}_{2}(t) \sin \left(\frac{3 \Omega t}{2}\right)\right] \\
& -\frac{\Omega^{3}}{8}\left[-A_{1}(t) \sin \left(\frac{\Omega t}{2}\right)+B_{1}(t) \cos \left(\frac{\Omega t}{2}\right)\right] \\
& -\frac{27 \Omega^{3}}{8}\left[-A_{2}(t) \sin \left(\frac{3 \Omega t}{2}\right)+B_{2}(t) \cos \left(\frac{3 \Omega t}{2}\right)\right] \\
+ & \left.\frac{\Omega^{2}}{4}+\Omega \delta\right)\left(\dot{A}_{1}(t) \cos \left(\frac{\Omega t}{2}\right)+\dot{B}_{1}(t) \sin \left(\frac{\Omega t}{2}\right)\right. \\
+ & \dot{A}_{2}(t) \cos \left(\frac{3 \Omega t}{2}\right)+\dot{B}_{2}(t) \sin \left(\frac{3 \Omega t}{2}\right) \\
+ & \frac{\Omega}{2}\left[-A_{1}(t) \sin \left(\frac{\Omega t}{2}\right)+B_{1}(t) \cos \left(\frac{\Omega t}{2}\right)\right] \\
+ & \left.\frac{3 \Omega}{2}\left[-A_{2}(t) \sin \left(\frac{3 \Omega t}{2}\right)+B_{2}(t) \cos \left(\frac{3 \Omega t}{2}\right)\right]\right) \\
+ & \frac{\Omega}{2}\left[-A_{1}(t) \sin \left(\frac{3 \Omega t}{2}\right)+A_{1}(t) \sin \left(\frac{\Omega t}{2}\right)\right. \\
+ & \left.\left.\frac{B_{2}}{2}(t) \cos \left(\frac{3 \Omega t}{2}\right)+B_{1}(t) \cos \left(\frac{3 \Omega t}{2}\right)+\dot{A}_{1}(t) \cos \left(\frac{\Omega t}{2}\right) \sin \left(\frac{\Omega t}{2}\right)+B_{2}(t) \cos \left(\frac{\Omega t}{2}\right)\right]\right) \\
+ & \dot{B}_{1}(t) \sin \left(\frac{3 \Omega t}{2}\right)-\dot{B}_{1}(t) \sin \left(\frac{\Omega t}{2}\right)
\end{aligned}
$$

$$
\begin{aligned}
& -\frac{\epsilon \Omega}{4}\left(A_{1}(t) \sin \left(\frac{\Omega t}{2}\right)+A_{1}(t) \sin \left(\frac{3 \Omega t}{2}\right)\right. \\
& +B_{1}(t) \cos \left(\frac{\Omega t}{2}\right)-B_{1}(t) \cos \left(\frac{3 \Omega t}{2}\right) \\
& \left.-A_{2}(t) \sin \left(\frac{\Omega t}{2}\right)+B_{2}(t) \cos \left(\frac{\Omega t}{2}\right)\right)=0
\end{aligned}
$$

\section{Appendix C}

In this appendix, we provide the calculational details behind the boundaries shown in Fig. 3. As mentioned in the following Eq. (38), the frequencies $\omega=\frac{\Omega}{6}$ and $\omega=\frac{\Omega}{2}$ are the ones that are capable of showing parametric resonance. Accordingly, we show in the appendix, how the perturbation theory and the KrylovBogoliubov technique can be used to obtain the boundaries of the unstable zones.

We first explore the regions near $\omega=\frac{\Omega}{6}$, writing $\omega=\frac{\Omega}{6}+\delta(\epsilon)$, so that Eq. (37) becomes

$$
\begin{aligned}
\dddot{S} & +10 \frac{\Omega^{2}}{36} \ddot{S}+9\left(\frac{\Omega^{2}}{36}\right)^{2} S+10 \frac{\Omega}{3} \delta \ddot{S} \\
& +36\left(\frac{\Omega}{6}\right)^{3} \delta S+10 \epsilon \cos (\Omega t) \ddot{S} \\
& +18 \frac{\Omega^{2}}{36} \epsilon \cos (\Omega t) S+9 \epsilon^{2} \cos ^{2}(\Omega t) S \\
& -10 \epsilon \Omega \sin (\Omega t) \dot{S}-3 \epsilon \Omega^{2} \cos (\Omega t) S=0
\end{aligned}
$$

With $\delta=\delta_{1} \epsilon+\delta_{2} \epsilon^{2}+\cdots$ as before, we have at the zeroth order

$$
\begin{aligned}
S_{0}= & A_{1} \cos \left(\frac{\Omega}{6} t\right)+B_{1} \sin \left(\frac{\Omega}{6} t\right)+A_{2} \cos \left(\frac{\Omega}{2} t\right) \\
& +B_{2} \sin \left(\frac{\Omega}{2} t\right)
\end{aligned}
$$

At $\mathcal{O}(\epsilon)$, we have

$$
\begin{aligned}
\dddot{S_{1}} & +10 \frac{\omega^{2}}{36} \ddot{S}_{1}+9\left(\frac{\omega^{2}}{36}\right)^{2} S_{1}=-10 \frac{\omega}{3} \delta_{1} \ddot{S}_{0} \\
& -36\left(\frac{\omega}{6}\right)^{3} \delta_{1} S_{0}-10 \cos (\Omega t) \ddot{S}_{0}-\frac{\omega^{2}}{2} \cos (\Omega t) S_{0} \\
& +10 \Omega \sin (\Omega t) \dot{S}_{0}+3 \Omega^{2} \cos (\Omega t) S_{0} \\
= & 10 \frac{\omega}{3} \delta_{1}\left[\frac{\Omega^{2}}{36} A_{1} \cos \left(\frac{\Omega}{6} t\right)+\frac{\Omega^{2}}{36} B_{1} \sin \left(\frac{\Omega}{6} t\right)\right. \\
& \left.+\frac{\Omega^{2}}{4} A_{2} \cos \left(\frac{\Omega}{2} t\right)+\frac{\Omega^{2}}{4} B_{2} \sin \left(\frac{\Omega}{2} t\right)\right] \\
& -36\left(\frac{\omega}{6}\right)^{3} \delta_{1}\left[A_{1} \cos \left(\frac{\Omega}{6} t\right)+B_{1} \sin \left(\frac{\Omega}{6} t\right)\right.
\end{aligned}
$$




$$
\begin{aligned}
& \left.+A_{2} \cos \left(\frac{\Omega}{2} t\right)+B_{2} \sin \left(\frac{\Omega}{2} t\right)\right] \\
& +10 \cos (\Omega t)\left[\frac{\Omega^{2}}{36} A_{1} \cos \left(\frac{\Omega}{6} t\right)+\frac{\Omega^{2}}{36} B_{1} \sin \left(\frac{\Omega}{6} t\right)\right. \\
& \left.+\frac{\Omega^{2}}{4} A_{2} \cos \left(\frac{\Omega}{2} t\right)+\frac{\Omega^{2}}{4} B_{2} \sin \left(\frac{\Omega}{2} t\right)\right] \\
& -\frac{\omega^{2}}{2} \cos (\Omega t)\left[A_{1} \cos \left(\frac{\Omega}{6} t\right)+B_{1} \sin \left(\frac{\Omega}{6} t\right)\right. \\
& \left.+A_{2} \cos \left(\frac{\Omega}{2} t\right)+B_{2} \sin \left(\frac{\Omega}{2} t\right)\right] \\
& +10 \Omega \sin (\Omega t)\left[-\frac{\Omega}{6} A_{1} \sin \left(\frac{\Omega}{6} t\right)+\frac{\Omega}{6} B_{1} \cos \left(\frac{\Omega}{6} t\right)\right. \\
& \left.-\frac{\Omega}{2} A_{2} \sin \left(\frac{\Omega}{2} t\right)+\frac{\Omega}{2} B_{2} \cos \left(\frac{\Omega}{2} t\right)\right] \\
& +3 \Omega^{2} \cos (\Omega t)\left[A_{1} \cos \left(\frac{\Omega}{6} t\right)+B_{1} \sin \left(\frac{\Omega}{6} t\right)\right. \\
& \left.+A_{2} \cos \left(\frac{\Omega}{2} t\right)+B_{2} \sin \left(\frac{\Omega}{2} t\right)\right]
\end{aligned}
$$

For a finite $S_{1}$ to exist, there can be no term in $\cos \left(\frac{\Omega}{6} t\right), \sin \left(\frac{\Omega}{6} t\right), \cos \left(\frac{\Omega}{2} t\right)$ and $\cos \left(\frac{\Omega}{2} t\right)$ on the r.h.s of Eq. (C.3). It is easy to check that the only term of this variety are the first two terms which are proportional to $\delta_{1}$ and hence for finiteness, we need $\delta_{1}=0$.

Carrying out the calculation to $\mathcal{O}\left(\epsilon^{2}\right)$ as done in Sect. 3 for $V$, we did not find any divergent region near $\omega=\frac{\Omega}{6}$, and hence, we look for possible divergent solution in the vicinity of $\omega=\frac{\Omega}{2}$. Writing $\omega=\frac{\Omega}{2}+$ $\delta_{1} \epsilon+\delta_{2} \epsilon^{2}+$.. we rewrite Eq. (37) to $\mathcal{O}(\epsilon)$ as,

$$
\begin{aligned}
\dddot{S} & +10 \frac{\Omega^{2}}{4} \ddot{S}+9\left(\frac{\Omega^{2}}{4}\right)^{2} S+10 \Omega \epsilon \delta_{1} \ddot{S} \\
& +36\left(\frac{\Omega}{2}\right)^{3} \delta_{1} \epsilon S+10 \epsilon \cos (\Omega t) \ddot{S}+18 \frac{\Omega^{2}}{4} \epsilon \cos (\Omega t) S \\
& -10 \epsilon \Omega \sin (\Omega t) \dot{S}-3 \epsilon \Omega^{2} \cos (\Omega t) S=0
\end{aligned}
$$

Expanding $S=S_{0}+\epsilon S_{1}+\cdots$, we have

$$
\begin{aligned}
S_{0}= & A_{1} \cos \left(\frac{\Omega}{2} t\right)+B_{1} \sin \left(\frac{\Omega}{2} t\right)+A_{2} \cos \left(\frac{3 \Omega}{2} t\right) \\
& +B_{2} \sin \left(\frac{3 \Omega}{2} t\right)
\end{aligned}
$$

At the next order, i.e., we get

$$
\begin{aligned}
\dddot{S}_{1} & +10 \frac{\Omega^{2}}{4} \ddot{S}_{1}+9\left(\frac{\Omega^{2}}{4}\right)^{2} S_{1}=-10 \Omega \delta_{1} \ddot{S}_{0} \\
& -\frac{9}{2} \Omega^{3} \delta_{1} S_{0}-10 \Omega^{2} \cos (\Omega t) \ddot{S_{0}}-\frac{9}{2} \Omega^{2} \cos (\Omega t) S_{0} \\
& +10 \Omega^{3} \sin (\Omega t) \dot{S_{0}}+3 \Omega^{4} \cos (\Omega t) S_{0}
\end{aligned}
$$

$$
\begin{aligned}
= & 10 \Omega \delta_{1}\left[\frac{\Omega^{2}}{4} A_{1} \cos \left(\frac{\Omega}{2} t\right)+\frac{\Omega^{2}}{4} B_{1} \sin \left(\frac{\Omega}{2} t\right)\right] \\
& -\frac{9}{2} \Omega^{3} \delta_{1}\left[A_{1} \cos \left(\frac{\Omega}{2} t\right)+B_{1} \sin \left(\frac{\Omega}{2} t\right)\right] \\
& +\frac{5}{8} \Omega^{4} \cos (\Omega t)\left[9\left(A_{2} \cos \left(\frac{3 \Omega}{2} t\right)+B_{2} \sin \left(\frac{3 \Omega}{2} t\right)\right)\right. \\
& \left.+\left(A_{1} \cos \left(\frac{\Omega}{2} t\right)+B_{1} \sin \left(\frac{\Omega}{2} t\right)\right)\right] \\
& +\frac{5}{4} \Omega^{4} \sin (\Omega t)\left[3\left(-A_{2} \sin \left(\frac{3 \Omega}{2} t\right)+B_{2} \cos \left(\frac{3 \Omega}{2} t\right)\right)\right. \\
& \left.+\left(-A_{1} \sin \left(\frac{\Omega}{2} t\right)+B_{1} \cos \left(\frac{\Omega}{2} t\right)\right)\right] \\
& -\frac{3}{8} \Omega^{4} \cos (\Omega t)\left[A_{1} \cos \left(\frac{\Omega}{2} t\right)+B_{1} \sin \left(\frac{\Omega}{2} t\right)\right. \\
& \left.+A_{2} \cos \left(\frac{3 \Omega}{2} t\right)+B_{2} \sin \left(\frac{3 \Omega}{2} t\right)\right]
\end{aligned}
$$

The resonance including terms on the right-hand side of Eq. (C.6) can be written as

$$
\begin{aligned}
& -2 \delta_{1}\left(A_{1} \cos \left(\frac{\Omega}{2} t\right)+B_{1} \sin \left(\frac{\Omega}{2} t\right)\right) \\
& +18 \delta_{1}\left(A_{2} \cos \left(\frac{3 \Omega}{2} t\right)+B_{2} \sin \left(\frac{3 \Omega}{2} t\right)\right) \\
& +\frac{\Omega}{16} \cos \left(\frac{\Omega}{2} t\right)\left(-8 A_{1}+12 A_{2}\right) \\
& +\frac{\Omega}{16} \sin \left(\frac{\Omega}{2} t\right)\left(8 B_{1}+12 B_{2}\right) \\
& +\frac{\Omega}{16} \cos \left(\frac{3 \Omega}{2} t\right)\left(12 A_{2}\right)+\frac{\Omega}{16} \sin \left(\frac{3 \Omega}{2} t\right)(
\end{aligned}
$$

Removal of each of the linearly independent terms leads to

$$
\begin{aligned}
& -2 \frac{\delta_{1}}{\Omega} A_{1}-\frac{A_{1}}{2}+3 \frac{A_{2}}{4}=0 \\
& -2 \frac{\delta_{1}}{\Omega} B_{1}-\frac{B_{1}}{2}+3 \frac{B_{2}}{4}=0 \\
& 18 \frac{\delta_{1}}{\Omega} A_{2}+\frac{3}{4} A_{1}=0 \\
& 18 \frac{\delta_{1}}{\Omega} B_{2}+\frac{3}{4} B_{1}=0
\end{aligned}
$$

The periodic solution $S(t)=A_{1} \cos \left(\frac{\Omega}{2} t\right)+A_{2}$ $\cos \left(\frac{3 \Omega}{2} t\right)$ occurs on the line obtained from consistency of $\frac{3}{4} A_{2}=\left(\frac{2 \delta_{1}}{\Omega}+\frac{1}{2}\right) A_{1}$ and $\frac{\delta_{1}}{\Omega} A_{2}+\frac{A_{1}}{24}=0$ which leads to $\delta_{1}=-\frac{\Omega}{8}$ and the solution $S(t)=B_{1} \sin \left(\frac{\Omega}{2} t\right)+$ $B_{2} \sin \left(\frac{3 \Omega}{2} t\right)$ exists on the line obtained from consistency of $\frac{3}{4} B_{2}=\left(-\frac{2 \delta_{1}}{\Omega}+\frac{1}{2}\right) B_{1}$ and $\frac{\delta_{1}}{\Omega} B_{2}-\frac{B_{1}}{24}=0$, which leads to $\delta_{1}=\frac{\Omega}{8}$. Around $\omega=\Omega / 2$, we have 
the periodic boundaries at Fig. 3

$$
\begin{aligned}
\omega= & \frac{\Omega}{2}-\frac{\epsilon \Omega}{8}+\mathcal{O}\left(\epsilon^{2}\right) \text { for } S=A_{1} \cos \left(\frac{\Omega}{2} t\right) \\
& +A_{2} \cos \left(\frac{3 \Omega}{2} t\right) \\
\omega= & \frac{\Omega}{2}+\frac{\epsilon \Omega}{8}+\mathcal{O}\left(\epsilon^{2}\right) \text { for } S=B_{1} \sin \left(\frac{\Omega}{2} t\right) \\
& +B_{2} \sin \left(\frac{3 \Omega}{2} t\right)
\end{aligned}
$$

These boundaries are shown blue shaded region in Fig. 3.

\section{References}

1. Jordan, D., Smith, P.: Ordinary Nonlinear Differential Equation, Chap. 9. Oxford University Press, Oxford (2007)

2. Landau, L.D., Lifshitz, E.M.: Mechanics. Peragmon Press Ltd, Oxford (1960). Chapter 6

3. Rand, R.H.: Lecture Notes on Nonlinear Vibrations. Cornell University (2014)

4. Nayfeh, A.H., Zavodney, L.D.: Experimental observation of amplitude-and phase-modulated responses of two internally coupled oscillators to a harmonic excitation. J. Appl. Mech. 55(3), 706-710 (1988)

5. Bountis, T.C., Mahmoud, G.M.: Synchronized periodic orbits in beam-beam interaction models of one and two spatial dimensions. Part. Accel. 22, 129-147 (1987)

6. Natsiavas, S.: On the dynamics of rings rotating with variable spin speed. Nonlinear Dyn. 7(3), 345-363 (1995)

7. Mahmoud, G.M.: Stability regions for coupled Hill's equations. Physica A Stat. Mech. Appl. 242(1-2), 239-249 (1997)

8. Landa, H., Drewsen, M., Reznik, B., Retzker, A.: Classical and quantum modes of coupled Mathieu equations. J. Phys. A Math. Theor. 45(45), 455305 (2012)

9. Lewis Jr., H.R., Riesenfeld, W.B.: An exact quantum theory of the time dependent harmonic oscillator and of a charged particle in a timedependent electromagnetic field. J. Math. Phys. 10(8), 1458-1473 (1969)

10. Younesian, D., Esmailzadeh, E., Sedaghati, R.: Existence of periodic solutions for the generalized form of Mathieu equation. Nonlinear Dyn. 39(4), 335-348 (2005)

11. Abraham, G.T., Chatterjee, A.: Approximate asymptotics for a nonlinear Mathieu equation using harmonic balance based averaging. Nonlinear Dyn. 31(4), 347-365 (2003)

12. Ng, L., Rand, R.: Bifurcations in a Mathieu equation with cubic nonlinearities. Chaos Solitons Fractals 14(2), 173-181 (2002)
13. Rand, R., Guennoun, K., Belhaq, M.: 2:2:1 Resonance in the quasiperiodic Mathieu equation. Nonlinear Dyn. 31(4), 367-374 (2003)

14. Insperger, T., Stépán, G.: Stability chart for the delayed Mathieu equation. Proc. R. Soc. Lond. A Math. Phys. Eng. Sci. 458(2024), 1989-1998 (2002)

15. Morrison, T.M., Rand, R.H.: 2: 1 Resonance in the delayed nonlinear Mathieu equation. Nonlinear Dyn. 50(1-2), 341352 (2007)

16. Xu, Y., Li, Y., Liu, D., Jia, W., Huang, H.: Responses of Duffing oscillator with fractional damping and random phase. Nonlinear Dyn. 73(3), 745-753 (2013)

17. Shen, Y.J., Wei, P., Yang, S.P.: 2: Primary resonance of fractional-order van der Pol oscillator. Nonlinear Dyn. 77(4), 1629-1642 (2014)

18. Wen, S., Shen, Y., Li, X., Yang, S., Xing, H.: 2: Dynamical analysis of fractional-order Mathieu equation. J. Vibroeng. 17(5), 2696-2709 (2015)

19. Erbe, L.H.: Third order linear differential equations with periodic coefficients. Proc. Am. Math. Soc. 64(2), 241-247 (1977)

20. Baesch, A.: On the explicit determination of certain solutions of periodic differential equations of higher order. Results Math. 29(1-2), 43-45 (1996)

21. Xiao, L.P.: Oscillation theorem for higher-order linear differential equations with periodic coefficients. J. Math. Anal. Appl. 376(2), 713-724 (2011)

22. Shimomura, S.: Oscillation results for $n$-th order linear differential equations with meromorphic periodic coefficients. Nagoya Math. J. 166, 55-82 (2002)

23. See e.g. Dittrich, W., Reuter, M.: Classical and Quantum Dynamics, vol. 20. Springer, Berlin (1994)

24. Brown, L.S.: Quantum motion in a Paul trap. Phys. Rev. Lett. 66(5), 527 (1991)

25. Glauber, R. J.: "Laser Manipulation of Atoms and Ions" Proceedings of the International School of Physics, "Enrico Fermi" volume 118, year, ed. by Arimondo, E., Philips, W. D., and Strumia, F. p-643 (1993)

26. Leibfried, D., Blatt, R., Monroe, C., Wineland, D.: Quantum dynamics of single trapped ions. Rev. Mod. Phys. 75(1), 281 (2003)

27. Hashemloo, A., Dion, C.M., Rahali, G.: Wave packet dynamics of an atomic ion in a Paul trap: approximations and stability. Int. J. Mod. Phys. C 27(02), 1650014 (2016)

28. Biswas, S., Chattopadhyay, R., Bhattacharjee, J.K.: Propagation of arbitrary initial wave packets in a quantum parametric oscillator: instability zones for higher order moments. Phys. Lett. A 382(18), 1202-1206 (2018)

29. Kovacic, I., Rand, R., Sah, S.M.: Mathieu's equation and its generalizations: overview of stability charts and their features. Appl. Mech. Rev. 70(2), 1020802 (2018)

Publisher's Note Springer Nature remains neutral with regard to jurisdictional claims in published maps and institutional affiliations. 